\title{
Pulmonary hypoplasia: lung weight and radial alveolar count as criteria of diagnosis
}

\author{
S. S. ASKENAZI AND M. PERLMAN
}

Hadassah Hospital, Jerusalem

SUMMARY A working definition of pulmonary hypoplasia $(\mathrm{PH})$ was established by retrospective assessment of lung growth both in recognised and hypothetical $\mathrm{PH}$-associated conditions. Lung weight: body weight ratios ( $\mathrm{LW}: \mathrm{BW}$ ) were calculated, and morphometry was determined by the radial alveolar count (RAC) (Emery and Mithal, 1960). Both parameters were reduced compared with those of normal controls in diaphragmatic hernia, anencephalus, anuric renal anomalies, chondrodystrophies, and osteogenesis inperfecta. Comparison of LW:BW ratio and RAC indicated that the RAC was the more reliable criterion of $\mathrm{PH}, \mathrm{LW}: \mathrm{BW}$ ratio of $\leq 0.012(67 \%$ of mean normal ratio) and/or RAC of $\leq 4.1$ (75\% of mean normal count) are suggested as diagnostic criteria of $\mathrm{PH}$. Evidence of PH was incidentally discovered in a number of clinically unsuspected cases and retrospectively clarified the clinical and radiological findings. Routine assessment of lung growth should be an essential part of the neonatal necropsy.

Pulmonary hypoplasia $(\mathrm{PH})$ is a poorly defined condition considered to be almost invariably secondary to other anomalies and is usually diagnosed in association with them; primary or isolated PH has not been reported (Reale and Esterly, 1973). PH is lethal within hours of birth in many instances of diaphragmatic hernia (Potter, 1961; Kitagawa et al., 1971; Reale and Esterly, 1973), anencephalus (Potter, 1961; Naeye and Blanc, 1971; Reale and Esterly, 1973), anuric renal anomalies with oligohydramnios (Potter, 1972; Reale and Esterly, 1973; Perlman and Levin, 1974; Thomas and Smith, 1974), and skeletal anomalies restricting thoracic volume (Finegold et al., 1971). There is however a spectrum of severity of $\mathrm{PH}$, often proportional to the severity of the underlying condition, and sublethal forms exist (Kohler and Babbitt, 1970; Chatrath et al., 1971; Perlman et al., 1975).

Because histological findings lack specificity (Potter, 1961; Reale and Esterly, 1973), diagnosis depends on detection of low lung weight in relation to body weight (low LW:BW ratio), or of low alveolar counts determined by morphometric methods (Reale and Esterly, 1973). Severe PH is easy to diagnose at necropsy from the low $\mathrm{LW}$ :BW ratio alone, but lesser or borderline PH is more difficult to

Neonatal Unit, Department of Paediatrics, Hadassah Hospital, Jerusalem

S. S. ASKENAZI, intern

M. PERLMAN, senior lecturer establish. There has been only one study of both $\mathrm{LW}: \mathrm{BW}$ ratio and morphometry in a substantial number of pathological cases (Reale and Esterly, 1973).

These diagnostic criteria of lung growth were studied in an attempt to establish a working definition of PH. Lungs of patients dying perinatally with $\mathrm{PH}-$ associated conditions were examined. In addition to these anomalies, neonates with other conditions in whom there were theoretical grounds to anticipate $\mathrm{PH}$ were examined.

\section{Materials and methods}

Material was obtained from the pathology departments of the Hadassah University Hospital, Jerusalem; Kaplan Hospital, Rehovot; and the Soroka Medical Centre, Beer-Sheva. About 200 liveborn and stillborn subjects were selected according to recognised $\mathrm{PH}$ associations (diaphragmatic hernia, anencephalus, anuric renal anomalies, chronic amniotic fluid leakage, and chondrodystrophies) and, in unprecedented cases, where PH was anticipated on theoretical grounds (osteogenesis imperfecta, hydrops fetalis). In addition, $\mathbf{P H}$ was identified incidentally in some patients who died during the study period because of a low $\mathrm{LW}: \mathrm{BW}$ ratio at necropsy (central nervous system anomalies, gross ascites due to long-standing meconium peritonitis). No patient with $\mathrm{PH}$ lived for more than 72 hours. 
'Normal' control liveborn and stillborn infants were selected randomly, but subjects with birthweights outside the 10th to 90th centile for gestational age were excluded. Patients with clinically diagnosed and pathological confirmed lung disease--such as hyaline membrane disease and meconium pneumonitis-or with congenital anomalies including heart defects other than those with $\mathbf{P H}$ associations were also excluded. In all control infants death resulted from either perinatal asphyxia or traumatic intracranial haemorrhage within 3 days of birth.

The following information was obtained from the necropsy record: sex, gestational age, body weight, lung weight, age at death, anatomic diagnoses, and cause of death. LW:BW ratios were computed.

Morphometry was performed by one of us (S.A.A.) at microscopical magnification of $\times 40$, using a modification of the method of Emery and Mithal (1960), without knowledge of the anatomical diagnoses or cause of death. Before beginning the study, experience of interobserver error was obtained with the help of Professor E. Rosenmann: the error was $< \pm 10 \%$. A line was dropped from the centre of each respiratory bronchiole (bronchioles only partially lined with epithelium) to the nearest connective tissue septum at right-angles to the epithelium. The number of alveolar septi (as distinct from the number of alveoli counted by Emery and Mithal) cut by this line were counted. At least 10 counts were made for each patient, mainly from 2-3 histological sections.

Mean values of $L W: B W$ ratios and of RACs and SDs of the mean were calculated for each diagnostic group. The significance of the difference of the means of each of the pathological groups compared with the control group was determined by Student's $t$ test. Subgroups of infants $<36$ weeks' gestation and $>35$ weeks' gestation were treated statistically in the same manner and compared.

\section{Results}

Of about 200 cases screened for study, 111 had adequate perinatal data and histological preparations suitable for morphometry. LW:BW ratios were not available in 12 cases. Mean gestational age and mean birthweight were similar in each group. The data for $\mathrm{LW}: \mathrm{BW}$ ratio and RAC for infants of gestational ages $<36$ weeks were compared with data for $>36$ weeks and there was no significant difference, so the total data were analysed.

With the exception of LW:BW ratios in anencephalics, LW:BW ratios and RAC were much reduced in each pathological group (Table). The results are illustrated graphically by plotting $\mathrm{LW}: \mathrm{BW}$ ratios against RAC (Figure). Lines were drawn to

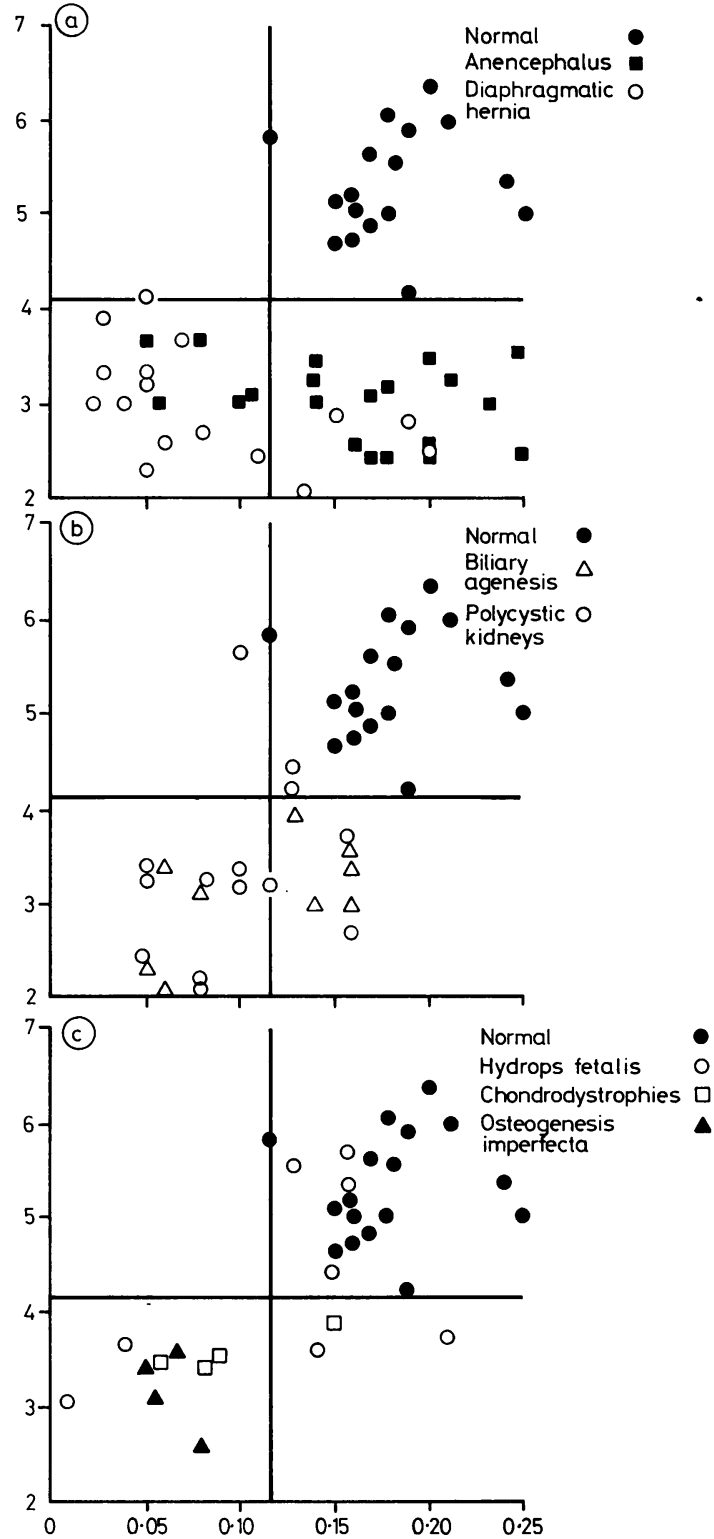

Figure Comparison of lung weight:body weight ratio and radial alveolar count for each aetiological category as indicated. Values for individual patients are plotted according to absolute values of $L W: B W$ ratio (abscissa, bottom), and mean RAC (ordinate, left). Additional horizontal and vertical lines, respectively, represent the values for the lower limits of normal for RAC and $L W: B W$ ratio.

indicate the lower limits of normal for each of the two parameters. The number of measurements below the normal range (mean -2 SDs) for each 
Table Lung weight; body weight ratios and radial alveolar counts in different aetiological categories

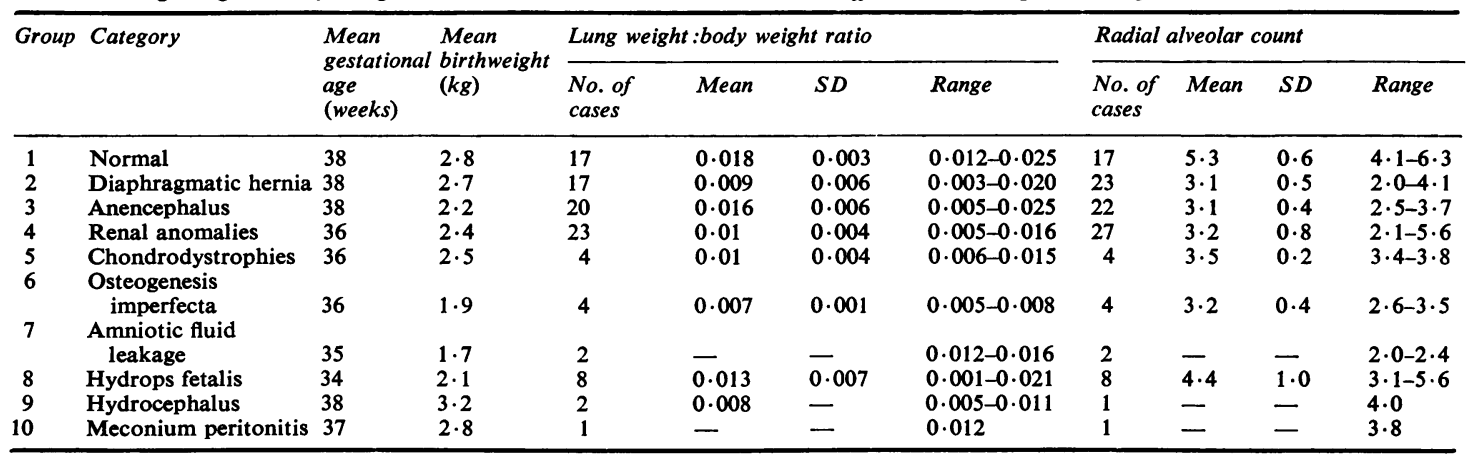

P value for the differences of the means of each of the groups 2-6 and of group 1 was at least $<0.01$ for LW:BW ratio and $<0.005$ for RAC.

group was greater for $\mathrm{LW}: \mathrm{BW}$ ratio than for RAC. The number of cases with values for both $L W: B W$ ratio and RAC within the normal range is also given. Complementary use of the two criteria failed to detect PH in only a very few cases.

\section{Discussion}

Pulmonary hypoplasia is diagnosed in $7 \cdot 8$ to $10.9 \%$ of neonatal necropsies and in about half of necropsied neonates with congenital anomalies (Driscoll and Smith, 1962; Pryse-Davies, 1972; Sultana et al., 1973), yet diagnostic criteria of $\mathrm{PH}$ have not been established. Whereas histological descriptions of PH have limited value (Potter, 1961; Reale and Esterly, 1973), the RAC provides a simple objective measurement of 'relative paucity of alveoli' or 'crowding of bronchial structures' which is unaffected by the state of expansion of the lungs (Emery and Mithal, 1960). At present $\mathrm{PH}$ is usually identified from associated anatomical diagnoses, such as those studied by ourselves. However, the diagnosis is often overlooked, especially when diagnostic associations are lacking. Moreover, a prejudiced diagnosis of $\mathbf{P H}$ may be made when anticipated from diagnostic associations.

Our results of $L W: B W$ ratios and RAC are similar to those of Emery and Mithal (1960) when differences in the method (see Materials and Methods) are accounted for (RAC alveolar count $=$ RAC alveolar septal count -1).

While we agree that typical histological changes of $\mathrm{PH}$ are due to quantitative rather than to qualitative factors (Reale and Esterly, 1973), we have reservations about anencephalus. We note a discrepancy between the LW:BW ratio and the RAC (Fig. 1a) which is only partially explained by a spuriously low body weight due to absence of the brain. With the exception of cases with associated thoracic skeletal anomalies (Potter, 1961), the pathogenesis of PH in anencephalus has never been satisfactorily explained. This inconsistency between $\mathrm{LW}: \mathrm{BW}$ ratio and RAC suggests a specific delay in alveolar development, possibly related to the hypothetical role of the pituitary in lung growth (Jain et al., 1973). Animal models of anencephalus produced at $0 \cdot 6-0 \cdot 7$ of gestation do not appear to have fewer air sacs (Blackburn et al., 1972; Meyrick et al., 1975) but the validity of the model for study of lung growth throughout gestation is questionable.

Necropsy studies of lung development in diaphragmatic hernia are not fully representative of this condition, as patients with less severe $\mathrm{PH}$ survive. In survivors there is a tendency towards progressive improvement postoperatively but normal lung function values may never be attained (Chatrath et al., 1971).

In the renal anomaly group the mean values for LW:BW ratio and the RAC were lower than those reported by Reale and Esterly (1973). This is explained by the fact that the latter study included unilateral renal anomalies, which are probably unassociated with PH (Perlman and Levin, 1974).

In our study we showed that $\mathbf{P H}$ is the probable cause of death in osteogenesis imperfecta congenita, presumably as a result of restriction of lung growth by the skeletal lesion, as occurs in chondrodystrophies (Finegold et al., 1971). PH was also demonstrated in some instances of hydrops fetalis, as reported by Chamberlain et al. (1977), and may have been caused by the thoracic space-occupying effects of large pleural effusions and raising of the diaphragm by tense ascites and gross hepatosplenomegaly. It is suggested that a similar mechanism exists that raises the diaphragm in $\mathrm{PH}$ associated with ascites in meconium peritonitis.

There is fairly good agreement of reported normal values of lung weights or $\mathrm{LW}: \mathrm{BW}$ ratios in different 
series (0·18-0.022) (Schultz et al., 1962; Potter, 1972; Reale and Esterly, 1973), but by excluding lungs with oedema and exudates, Emery and Mithal (1969) arrived at a mean $\mathrm{LW}: \mathrm{BW}$ ratio of 0.013. Normal lung weight is greatly influenced by postnatal absorption of lung fluid (Aherne and Dawkins, 1964). In spite of these limitations, an LW:BW ratio $<0.012$ suggested $\mathrm{PH}$ at all gestational ages in our material. This value was outside the range of our normal controls but was higher than half the normal mean (0.009) arbitrarily suggested by Potter (1972) as a criterion of $\mathbf{P H}$. In those instances of $\mathbf{P H}$ discovered incidentally, a $\mathrm{LW}: \mathrm{BW}$ ratio $<0.012$ led to confirmation of the diagnosis of PH by RAC, and in the light of this diagnosis the clinical and radiological manifestations were retrospectively made more comprehensible.

Data on the number of measurements of each parameter that came within the normal range (Figure) suggest that in each case, the RAC provides a clearer definition between $\mathrm{PH}$ and normal subjects than the LW:BW ratio. It should be pointed out that the difference between the mean normal RAC $(5 \cdot 3)$ and lower limit of normal $(4 \cdot 1)$ becomes greater when these values are raised to the third power (149 v. 69) in order to produce a 3-dimensional measure (i.e. number of alveoli in terminal respiratory units) from the single dimension that the RAC represents (Emery and Mithal, 1960).

We suggest the following guidelines for the diagnosis of PH. LW:BW ratio $<0 \cdot 009$, $\mathrm{PH}$ very likely, RAC not obligatory. LW:BW ratio $0 \cdot 010-0 \cdot 012$, $\mathrm{PH}$ probable, RAC indicated for confirmation of diagnosis. LW:BW ratio $0 \cdot 013-0 \cdot 017$, $\mathrm{PH}$ possible, $\mathrm{RAC}$ desirable. $\mathrm{LW}: \mathrm{BW}$ ratio $>0 \cdot 018$, $\mathrm{PH}$ unlikely, and RAC probably not indicated. Where RAC is estimated, a value $<75 \%$ of the mean normal value (equivalent to $43 \%$ of cubed value) for that laboratory probably indicates $\mathbf{P H}$.

PH is rarely diagnosed in living patients but there is increasing awareness of this condition (Renert et al., 1972; Leonidas et al., 1975). The existence of a spectrum of lung hypoplasia has been recognised but this is not shown in a necropsy study owing to lack of representation of less severe or borderline PH patients who are excluded from study by virtue of their survival (De Lorimier et al., 1967; Burkle and Bravo, 1969; Potter, 1972; Perlman et al., 1975). Finally, the role of impaired lung growth in other neonatal pulmonary conditions-such as hydrops fetalis and even hyaline membrane disease-has not been fully explored.

We thank Professor E. Rosenmann for interpreting lung histology, and Professor B. Czernobilsky and Professor M. Sachs for access to material.

\section{References}

Aherne, W., and Dawkins, M. J. R. (1964). The removal of fluid from the pulmonary airways after birth in the rabbit, and the effect on this of prematurity and prenatal hypoxia. Biologia Neonatorum, 7, 214-229.

Blackburn, W. R., Travers, H., and Potter, D. M. (1972). The role of the pituitary-adrenal-thyroid axes in lung differentiation. I. Studies of the cytology and physical properties of anencephalic fetal rat lung. Laboratory Investigation, 26, 306-318.

Burkle, F. M., Jr, and Bravo, A. J. (1969). Asphyxiating thoracic dystrophy malformation of the newborn. Clinical Pediatrics, 8, 165-170.

Chamberlain, D., Hislop, A., Hey, E., and Reid, L. (1977). Pulmonary hypoplasia in babies with severe rhesus isoimmunisation: a quantitative study. Journal of Pathology, 122, 43-52.

Chatrath, R. R., El Shafie, M., and Jones, R. S. (1971). Fate of hypoplastic lungs after repair of congenital diaphragmatic hernia. Archives of Disease in Childhood, 46, 633-635.

De Lorimier, A. A., Tierney, D. F., and Parker, H. R. (1967). Hypoplastic lungs in fetal lambs with surgically produced congenital diaphragmatic hernia. Surgery, 62, 12-17.

Driscoll, S. G., and Smith, C. A. (1962). Neonatal pulmonary disorders. Pediatric Clinics of North America, 9, 325-352.

Emery, J. L., and Mithal, A. (1960). The number of alveoli in the terminal respiratory unit of man. Archives of Disease in Childhood, 35, 544-547.

Emery, J. L., and Mithal, A. (1969). In The Anatomy of the Developing Lung, pp. 203-205. Edited by J. Emery. Heinemann Medical: London.

Finegold, J. J., Katzew, H., Brohon, N., and Becker, M. H. (1971). Lung structure in thoracic dystrophy. American Journal of Diseases of Children, 122, 153-159.

Jain, B. P., Brody, J. S., and Fisher, A. B. (1973). The small lung in hypopituitarism. American Review of Respiratory Diseases, 108, 49-55.

Kitagawa, M., Hislop, A., Boyden, E. A., and Reid, L. (1971). Lung hypoplasia in congenital diaphragmatic hernia. A quantitative study of airway, artery, and alveolar development. British Journal of Surgery, 58, 342-346.

Kohler, E., and Babbitt, D. P. (1970). Dystrophic thoraces and infantile asphyxia. Radiology, 94, 55-62.

Leonidas, J. C., Fellows, R. A., Hall, R. T., Rhodes, P. G., and Beatty, E. C. (1975). Value of chest radiography in the diagnosis of Potter's syndrome at birth. American Journal of Roentgenology, Radium Therapy, and Nuclear Medicine, 123, 716-723.

Meyrick, B., Bearer, J. G., Cobb, A. G., Monkhouse, C. R., and Reid, A. (1975). The effect of in utero decapitation on the morphological and physiological development of the fetal rabbit lung. Journal of Anatomy, 119, 517-535.

Naeye, R. L., and Blanc, W. A. (1971). Organ and body growth in anencephaly. Archives of Pathology, 91, 140147.

Perlman, M., and Levin, M. (1974). Fetal pulmonary hypoplasia, anuria, and oligohydramnios. American Journal of Obstetrics and Gynecology, 118, 1119-1123.

Perlman, M., Williams, J., and Hirsch, M. (1975). Neonatal pulmonary hypoplasia after prolonged leakage of amniotic fluid. Archives of Disease in Childhood, 51, 349-353.

Potter, E. L. (1961). Pathology of the Fetus and Infant, second edition, pp. 301-302. Year Book Medical Publishers: Chicago.

Potter, E. L. (1972). Normal and Abnormal Development of the Kidney, p. 97. Year Book Medical Publishers: Chicago.

Pryse-Davies, J. (1972). Pathology of the perinatal lung. Proceedings of the Royal Society of Medicine, 65, 823-824. 
Reale, F. R., and Esterly, J. R. (1973). Pulmonary hypoplasia -a morphometric study of the lungs of infants with diaphragmatic hernia, anencephalus, and renal malformation. Pediatrics, 51, 91-96.

Renert, W. A., Berdon, W. E., Baker, D. H., and Rose, J. C. (1972). Urological malformations of the fetus and infant: relation to neonatal pneumomediastinum and pneumothorax (air block). Radiology, 105, 97-105.

Schultz, D. M., Giordano, D. A., and Schultz, D. H. (1962). Weights of organs of fetuses and infants. Archives of Pathology, 74, 244-250.

Sultana, Z., Talib, V. H., Patil, S. O., Deshpande, M. S., and Sharina, K. D. (1973). Hypoplasia of the lung in the new- born-an autopsy study. Indian Journal of Pediatrics, 40, 419-421.

Thomas, I. T., and Smith, D. W. (1974). Oligohydramnios, cause of the nonrenal features of Potter's syndrome, including pulmonary hypoplasia. Journal of Pediatrics, 84, 811-815.

Correspondence to Dr Max Perlman, Department of Pediatrics, Children's Memorial Hospital, University of Oklahoma Health Sciences Center, POB 26901, Oklahoma City, Oklahoma 73190, USA.

Received 5 December 1978 